\title{
Molecular dynamics simulation of collision operator eigenvalues
}

\author{
Erich D. Gust and L. E. Reichl \\ Center for Complex Quantum Systems and Department of Physics, The University of Texas at Austin, Austin, Texas 78712, USA
}

(Received 21 October 2008; revised manuscript received 15 January 2009; published 16 March 2009)

\begin{abstract}
We simulate numerically the time evolution of 1000 interacting hard spheres in a finite box with periodic boundary conditions, and repeat the simulations many times for a selected random distribution of initial conditions. We use the resulting data to compute, directly, the smallest nonzero eigenvalue of the collision operator for this gas. We also give exact expressions for the transport coefficients and compare them to approximate expressions commonly seen in the literature.
\end{abstract}

DOI: 10.1103/PhysRevE.79.031202

PACS number(s): 51.10.+y, 51.20.+d, 02.70.Ns, 51.90.+r

\section{INTRODUCTION}

The decay to equilibrium of gases driven out of equilibrium, by stirring or selective heating by laser radiation, is governed by collision processes at the microscopic level. The effect of these collision processes on the macroscopic phase space distribution of the gas is contained in the collision operators that appear naturally in the kinetic equations that govern the time evolution of the gas as it approaches its equilibrium state. Linearizing the kinetic equation admits analysis in terms of linear collision operators. The eigenvalues of these linearized collision operators determine the rate at which a gas decays to equilibrium and the eigenvectors determine the modes of decay.

The eigenvalue problem for a Maxwell gas was solved by Wang Chang and Uhlenbeck [1]. The eigenvectors of the Maxwell gas can be used as an expansion basis for the linearized collision operator for a hard-sphere gas [2] and we follow this approach. In general, the eigenvalue spectrum may contain continuous regions as well as discrete values [3]. For hard spheres, the discrete eigenvalues accumulate at a limit point, making the higher-order discrete eigenvalues difficult to calculate [4]. Since we measure only the first few eigenvalues, the expansion method is sufficient.

The eigenvalue expansion has also been used to model decay processes in more complex situations such as the relaxation of light-induced changes of velocity distribution tracer atoms immersed in a light insensitive buffer gas [5-7], and the effect of collisions on spectral line shapes [8]. The collision operator for a gas also determines the value of its transport coefficients.

In the sections below, we measure directly the decay rate of the longest lived eigenmode (of the collision operator) for a hard-sphere gas, using data from a molecular dynamics simulation of 1000 hard spheres in a box. We begin in Sec. II by deriving the collision matrix for a hard-sphere gas and for a Maxwell gas since the eigenvectors of the collision matrix for a Maxwell gas are used in the hard-sphere derivation. In Sec. III, we give the results of the numerical simulation of 1000 hard spheres, initially held out of equilibrium and then allowed to decay to equilibrium using Newtonian mechanics. In Sec. IV we give exact expressions for the transport coefficients and, in Sec. V, we make some concluding remarks.

\section{DECAY RATES}

If the momentum distribution of a dilute gas of particles of diameter $\sigma$ and mass $m$ is driven out of equilibrium, then after a very short time its decay back to equilibrium will be governed by the single particle phase space distribution function $f(\mathbf{v}, t)$, where $\mathbf{v}$ is the velocity. The time evolution of this function is governed by the Boltzmann equation [1,9-12],

$$
\begin{aligned}
\frac{\partial f_{1}}{\partial t}= & \int d \mathbf{v}_{2} d \mathbf{v}_{3} d \mathbf{v}_{4} I(u, \theta) \delta^{3}\left(\frac{\mathbf{v}_{1}+\mathbf{v}_{2}}{2}-\frac{\mathbf{v}_{3}+\mathbf{v}_{4}}{2}\right) \\
& \times \delta\left(v_{1}^{2}+v_{2}^{2}-v_{3}^{2}-v_{4}^{2}\right)\left(f_{3} f_{4}-f_{1} f_{2}\right),
\end{aligned}
$$

where $I(u, \theta)$ is the differential scattering cross section for a binary interaction between two particles, $u$ is the magnitude of the relative velocity between two colliding particles, $\theta$ is the scattering angle, and $f_{1}=f\left(\mathbf{v}_{1}, t\right)$. The Boltzmann equation is used to model the effects of gas collisions under a variety of circumstances and to compute microscopic expressions for transport coefficients in gases.

For small amplitude deviations from equilibrium, the Boltzmann equation can be linearized and becomes an eigenvalue equation for the characteristic decay rates which determine the rate of relaxation of the gas to its equilibrium distribution which, for a simple gas, is the Maxwell-Boltzmann distribution. Let us note that the Maxwell-Boltzmann distribution can be written in the form $f^{0}(\mathbf{v})=\frac{n_{0}}{v_{T}^{3} \pi^{3 / 2}} e^{-c^{2}}$, where $n_{0}$ $=N / V, N$ is the number of particles, $V$ is the volume, $\mathbf{c}=\frac{\mathbf{v}}{v_{T}}$ is a dimensionless velocity coordinate, and $v_{T}=\sqrt{\frac{2 k_{B} T}{m}}$ represents the most probable velocity in equilibrium. Small deviations from equilibrium can be written us $f(\mathbf{v})=f^{0}(\mathbf{v})[1$ $+g(\mathbf{c})]$, where the dimensionless function $g(\mathbf{c})$ represents perturbations about equilibrium. We shall assume that $g(\mathbf{c})$ $\ll 1$ and, as the system relaxes, $g(\mathbf{c}) \rightarrow 0$.

If we keep terms linear in $g(\mathbf{c})$, the Boltzmann equation can be written as an evolution equation for $g(\mathbf{c})$, namely

$$
\frac{d g\left(\mathbf{c}_{1}\right)}{d t}=\int d \mathbf{c}_{5} e^{-c_{5}^{2}} C\left(\mathbf{c}_{1}, \mathbf{c}_{5}\right) g\left(\mathbf{c}_{5}\right),
$$

where

$$
\begin{aligned}
C\left(\mathbf{c}_{1}, \mathbf{c}_{5}\right)= & n_{0} v_{T} \frac{8 e^{c_{5}^{2}}}{\pi^{3 / 2}} \int d \mathbf{c}_{2} d \mathbf{c}_{3} d \mathbf{c}_{4} I\left(u^{\prime}, \theta\right) \delta^{3}\left(\mathbf{c}_{1}+\mathbf{c}_{2}-\mathbf{c}_{3}-\mathbf{c}_{4}\right) \\
& \times \delta\left(c_{1}^{2}+c_{2}^{2}-c_{3}^{2}-c_{4}^{2}\right) e^{-c_{2}^{2}}\left[\delta^{3}\left(\mathbf{c}_{3}-\mathbf{c}_{5}\right)\right. \\
& \left.+\delta^{3}\left(\mathbf{c}_{4}-\mathbf{c}_{5}\right)-\delta^{3}\left(\mathbf{c}_{1}-\mathbf{c}_{5}\right)-\delta^{3}\left(\mathbf{c}_{2}-\mathbf{c}_{5}\right)\right] .
\end{aligned}
$$

The function $C\left(\mathbf{c}_{1}, \mathbf{c}_{5}\right)$ is called the collision kernel. Explicit 
calculation shows that it is symmetric under interchange of $\mathbf{c}_{1}$ and $\mathbf{c}_{5}$ (see Appendix A). The collision kernel conserves the momentum, kinetic energy, and number of colliding particles. As we will see, its eigenvalues are either zero (due to conservation laws) or negative allowing decay of the gas to thermodynamic equilibrium.

The linearized Boltzmann equation given in Eqs. (2) and (3) can be converted into a matrix equation whose evolution is governed by a "collision matrix." The eigenvalues of the collision matrix give the characteristic decay rates of perturbations in the gas, and the inverse of the collision matrix determines the transport coefficients. In order to obtain the matrix Boltzmann equation, we introduce the Burnett functions $[1,9,12]$,

$$
\chi_{n, l, m}(\mathbf{c})=\sqrt{\frac{2 n !}{\left(n+l+\frac{1}{2}\right) !}} L_{n}^{l+(1 / 2)}\left(c^{2}\right) c^{l} Y_{l}^{m}(\hat{\mathbf{c}}),
$$

where $L_{n}^{l}\left(c^{2}\right)$ is a Laguerre polynomial and $Y_{l}^{m}(\hat{\mathbf{c}})$ is a spherical harmonic. The Burnett functions are orthonormal over c with weighting function $e^{-c^{2}}$. We can expand the functions $g(\mathbf{c})$ in terms of the Burnett functions,

$$
g(\mathbf{c})=\sum_{n=0}^{\infty} \sum_{l=0}^{\infty} \sum_{m=-l}^{l} g_{n, l, m} \chi_{n, l, m}(\mathbf{c}),
$$

which yields $g_{n, l, m}=\int d \mathbf{c} e^{-c^{2}} g(\mathbf{c}) \chi_{n, l, m}^{*}(\mathbf{c})$. The linearized Boltzmann equation then takes the form

$$
\frac{d g_{n, l, m}}{d t}=\sum_{n^{\prime} l^{\prime} m^{\prime}} C_{n^{\prime}, l^{\prime}, m^{\prime}}^{n, l, m} g_{n^{\prime}, l^{\prime}, m^{\prime}}
$$

where

$$
C_{n^{\prime}, l^{\prime}, m^{\prime}}^{n, l, m}=\int d \mathbf{c}_{1} d \mathbf{c}_{5} e^{-c_{1}^{2}-c_{5}^{2}} \chi_{n, l, m}^{*}\left(\mathbf{c}_{1}\right) C\left(\mathbf{c}_{1}, \mathbf{c}_{5}\right) \chi_{n^{\prime}, l^{\prime}, m^{\prime}}\left(\mathbf{c}_{5}\right) .
$$

The function $C\left(\mathbf{c}_{1}, \mathbf{c}_{5}\right)$ is a scalar quantity and can only depend on $\left|\mathbf{c}_{1}\right|,\left|\mathbf{c}_{5}\right|$, and $\hat{\mathbf{c}}_{1} \cdot \hat{\mathbf{c}}_{5}$. This fact results in $C$ being diagonal in $l$ and $m$ and independent of $m$ [1] so that

$$
C_{n^{\prime}, l^{\prime}, m^{\prime}}^{n, l, m}=C_{n, n^{\prime}}^{l} \delta_{l, l^{\prime}} \delta_{m, m^{\prime}} .
$$

Thus for each value of $l$ and $m$, we have a independent system and we are left with a simple matrix equation of the form

$$
\frac{d g_{n, l, m}}{d t}=\sum_{n^{\prime}=0}^{\infty} C_{n, n^{\prime}}^{l} g_{n^{\prime}, l, m} .
$$

The Boltzmann equation describes decay to an equilibrium state while conserving five quantities: Particle number, three components of momentum and energy. Thus we expect that the collision matrix will have five zero eigenvalues, and that all other eigenvalues will be negative.

Explicit calculation of the matrix elements $C_{n, n^{\prime}}^{l}$ requires knowledge of the scattering cross section $I(u, \theta)$. For problems where $I(u, \theta)$ is unknown, one may assume a phenomenological collision kernel with undetermined parameters
$[7,8,13,14]$. Comparing the resulting eigenvalues and transport coefficients to experimental results can then determine the parameters of the assumed collision kernel.

The general procedure for computing matrix elements $C_{n, n^{\prime}}^{l}$ involves use of a generating function, which is obtained as follows. First introduce a matrix $M_{n, n^{\prime}}^{l}$ which is defined

$$
\begin{aligned}
M_{n, n^{\prime}}^{l}= & \int d \mathbf{c}_{1} d \mathbf{c}_{5} e^{-c_{1}^{2}-c_{5}^{2}} C\left(\mathbf{c}_{1}, \mathbf{c}_{5}\right) c_{1}^{l} c_{5}^{l} L_{n}^{l+(1 / 2)}\left(c_{1}^{2}\right) \\
& \times L_{n^{\prime}}^{l+(1 / 2)}\left(c_{5}^{2}\right) P_{l}\left(\hat{\mathbf{c}}_{1} \cdot \hat{\mathbf{c}}_{5}\right),
\end{aligned}
$$

so that

$$
C_{n, n^{\prime}}^{l}=\frac{1}{4 \pi} \sqrt{\frac{4 n ! n^{\prime} !}{\Gamma\left(n+l+\frac{3}{2}\right) \Gamma\left(n^{\prime}+l+\frac{3}{2}\right)}} M_{n, n^{\prime}}^{l} .
$$

The spherical harmonic addition theorem $\sum_{m=-l}^{l} Y_{l}^{m *}\left(\hat{\mathbf{c}}_{1}\right) Y_{l}^{m}\left(\hat{\mathbf{c}}_{5}\right)=\frac{2 l+1}{4 \pi} P_{l}\left(\hat{\mathbf{c}}_{1} \cdot \hat{\mathbf{c}}_{5}\right)$ has been used to eliminate explicit dependence on $m$. The generating function for $M_{n, n^{\prime}}^{l}$ can be written as

$$
M(x, y ; t)=\sum_{n=0}^{\infty} \sum_{n^{\prime}=0}^{\infty} \sum_{l=0}^{\infty} M_{n, n^{\prime}}^{l} x^{n} y^{n^{\prime}} \frac{t^{l}}{l !} .
$$

Using the identities

$$
\sum_{n=0}^{\infty} L_{n}^{k}\left(c^{2}\right) x^{n}=\frac{1}{(1-x)^{k+1}} e^{-x c^{2} /(1-x)}
$$

and

$$
\sum_{l=0}^{\infty} P_{l}(\hat{\mathbf{a}} \cdot \hat{\mathbf{b}}) \frac{t^{l}}{l !}=\frac{1}{2 \pi} e^{t \hat{\mathbf{a}} \cdot \hat{\mathbf{b}}} \int_{0}^{2 \pi} e^{i t|\hat{\mathbf{a}} \times \hat{\mathbf{b}}| \cos \phi} d \phi
$$

for the Laguerre and Legendre polynomials, we obtain

$$
\begin{aligned}
M(x, y ; t)= & \frac{1}{2 \pi[(1-x)(1-y)]^{3 / 2}} \\
& \times \int d \mathbf{c}_{1} d \mathbf{c}_{5} e^{-c_{1}^{2}-c_{5}^{2}} C\left(\mathbf{c}_{1}, \mathbf{c}_{5}\right) \\
& \times e^{-x c_{1}^{2} /(1-x)-y c_{5}^{2} /(1-y)+t \mathbf{c}_{1} \cdot \mathbf{c}_{5} /[(1-x)(1-y)]} \\
& \times \int_{0}^{2 \pi} d \phi e^{i t\left|\mathbf{c}_{1} \times \mathbf{c}_{5}\right| \cos \phi /[(1-x)(1-y)]} .
\end{aligned}
$$

For some choices of interparticle potential, the integrals in Eq. (14) can be done analytically. The generating function $M(x, y, t)$ can be expanded in a power series in parameters $x$, $y$ and $t$. The coefficient of the term $x^{n} y^{n^{\prime}} t^{l}$ is equal to $M_{n, n^{\prime}}^{l} / l$ !. Once we know $M_{n, n^{\prime}}^{l}$, we can use Eq. (11) to obtain $C_{n, n^{\prime}}^{l}$. Below we give expressions for $C_{n, n^{\prime}}^{l}$ for a hard-sphere gas and for a gas of Maxwell particles.

\section{A. Hard-sphere gas}

For hard spheres, the differential scattering cross section is $I(u, \theta)=\pi \sigma^{2}$, where $\sigma$ is the diameter of the hard spheres. The collision matrix is given by 


$$
C_{n, n^{\prime}}^{l}=\frac{1}{\tau_{\mathrm{HS}}} \sqrt{\frac{n ! n^{\prime} !}{8 \Gamma\left(n+l+\frac{3}{2}\right) \Gamma\left(n^{\prime}+l+\frac{3}{2}\right)}} \sum_{j=0}^{n, n^{\prime}} \sum_{k=0}^{l} \frac{\Gamma\left(-\frac{1}{2}+n+n^{\prime}+l-2 j-k\right) l !}{(n-j) !\left(n^{\prime}-j\right) !(l-k) ! 2^{n+n^{\prime}+l-2 j-k}} B_{j}^{k},
$$

where $B_{j}^{k}=\frac{(j+k+1) !}{j ! k !}+\delta_{j 0} \delta_{k 0}-2 \frac{(2 j+k+1) !}{(2 j+1) ! k ! 2^{k}}$ and $\frac{1}{\tau_{\mathrm{HS}}}=n_{0} \pi \sigma^{2} \sqrt{\frac{8 k_{B} T}{\pi m}}$. More details concerning the derivation of Eq. (15) are given in Appendix A.

The matrix formed by the quantities $C_{n, n^{\prime}}^{l}$ is block diagonal in $l$. Let us introduce the matrix $\mathbf{C}^{l}$ such that $C_{n, n^{\prime}}^{l}=\left[\mathbf{C}_{n, n^{\prime}}\right.$. If we use Eq. (15), the matrix $\mathbf{C}^{0}$ is given by

$$
\mathbf{C}^{0}=\frac{1}{\tau_{\mathrm{HS}}}\left(\begin{array}{cccccc}
0 & 0 & 0 & 0 & 0 & \cdots \\
0 & 0 & 0 & 0 & 0 & \cdots \\
0 & 0 & -0.754247 & 0.174574 & 0.0205738 & \cdots \\
0 & 0 & 0.174574 & -1.25259 & 0.319048 & \cdots \\
0 & 0 & 0.0205738 & 0.319048 & -1.64374 & \cdots \\
\vdots & \vdots & \vdots & \vdots & \vdots & \ddots
\end{array}\right),
$$

We find eigenvalues of $\mathbf{C}^{0}$ numerically using the first 200 rows and columns. They are

$$
\lambda_{\mathrm{HS}}^{0}=\frac{1}{\tau_{\mathrm{HS}}}(0, \quad 0, \quad-0.67123, \quad-0.91157, \quad-0.98206, \quad \cdots, \quad-1) .
$$

It is well known that for hard spheres, all discrete eigenvalues are greater than $-\frac{1}{\tau_{\mathrm{HS}}}[2,4,15]$ and accumulate to the value $\lambda_{\mathrm{HS}}^{\mathbf{0}}=-\frac{1}{\tau_{\mathrm{HS}}}$. Our results are consistent with that observation. Only the first few eigenvalues are relevant for our hard-sphere simulation. The contributions to $C_{n n^{\prime}}^{l}$ coming from $l=0$ represent perturbations that only depend on the magnitude of the velocity. The two zero eigenvalues represent conservation of particles and conservation of energy.

For $l=1$, the collision matrix is

$$
\mathbf{C}^{1}=\frac{1}{\tau_{\mathrm{HS}}}\left(\begin{array}{cccccc}
0 & 0 & 0 & 0 & \cdots & \\
0 & -0.754247 & 0.142539 & 0.0145479 & \cdots & \\
0 & 0.142539 & -1.21218 & 0.283177 & \cdots & \\
0 & 0.0145479 & 0.283177 & -1.58734 & \cdots & \\
\vdots & \vdots & \vdots & \vdots & \vdots & \ddots
\end{array}\right),
$$

and its eigenvalues computed from the first 200 rows and columns are

$$
\begin{aligned}
\lambda_{\mathbf{H S}}^{1}= & \frac{1}{\tau_{\mathrm{HS}}}(0, \quad-0.69503, \quad-0.92114, \\
& -0.98581, \quad \cdots, \quad-1) .
\end{aligned}
$$

The single zero eigenvalue represents conservation of velocity in the $x, y$, and $z$ directions. It contains information about these three independent components of velocity because $l$ $=1$ represents perturbations that are proportional to $\mathbf{c}$ and otherwise only depend on the magnitude of $c$. The $l=1$ matrix actually represents three identical matrices with $m=-1$, $m=0$, and $m=1$ since the matrix elements are independent of $m$. This brings the total conserved quantities to five, the number we expect to have for spherically symmetric particles.

Perturbations that contain combinations such as $c_{x} c_{y}$ are represented by the $l=2$ matrix. The eigenvalues of $\mathbf{C}^{2}$ are given by

$$
\lambda_{\mathrm{HS}}^{2}=\frac{1}{\tau_{\mathrm{HS}}}(-0.95840, \quad-0.99505, \quad \cdots,-1) .
$$

The matrix $\mathbf{C}^{2}$ has no zero eigenvalues. In contrast, the diagonal elements of the matrix $\mathbf{C}^{2}$ are

$$
\begin{aligned}
& \frac{1}{\tau_{\mathrm{HS}}}(-1.13137, \quad-1.38054, \quad-1.66801, \\
& -1.94575, \quad-2.20563, \quad \cdots),
\end{aligned}
$$

showing that the off-diagonal elements have substantial influence in slowing the decay rates. For $l \geqslant 3$ it appears that no discrete eigenvalues exist $[2,4]$.

\section{B. Maxwell gas}

For a Maxwell gas, the interparticle potential is given by $V(r)=V_{4}\left(\frac{a}{r}\right)^{4}$ and the collision cross section for particles of mass $m$ is given by $I(u, \theta)=\frac{a^{2}}{8} \sqrt{\frac{2 V_{4}}{m}} \frac{1}{u} F(\theta)$, where 


$$
\begin{aligned}
F(\theta) & =\frac{4}{\sin \theta} \frac{\sqrt{\cos 2 \psi}}{\sin 2 \psi\left[\cos 2 \psi E(\sin \psi)-\cos ^{2} \psi K(\sin \psi)\right]} \\
& =\frac{2}{\sin \theta \sin ^{2} 2 \psi}\left(\frac{d \theta}{d \psi}\right)^{-1} .
\end{aligned}
$$

The angle $\psi$ is defined implicitly by the equation $\theta(\psi)=\pi$ $-2 \sqrt{\cos 2 \psi} K(\sin \psi) . K$ and $E$ are complete elliptic integrals of the first and second kinds, respectively. Though this cross section is complicated, the $\frac{1}{u}$ dependence results in $\chi_{n, l, m}$ being the exact eigenfunctions of the collision operator [1] for the Maxwell gas. The collision matrix $C_{n, n^{\prime}}^{l}$ is therefore diagonal and the eigenvalues can be written as

$$
\lambda_{n}^{l}=\frac{2 \pi}{\tau_{M}} \int_{0}^{\pi} G_{n}^{l}(\theta) F(\theta) \sin \theta d \theta,
$$

where

$$
\begin{aligned}
G_{n}^{l}(\theta)= & \cos ^{2 n+l}\left(\frac{\theta}{2}\right) P_{l}\left(\cos \frac{\theta}{2}\right)+\sin ^{2 n+l}\left(\frac{\theta}{2}\right) P_{l}\left(\sin \frac{\theta}{2}\right)-1 \\
& -\delta_{n 0} \delta_{l 0}
\end{aligned}
$$

and $\frac{1}{\tau_{M}}=n_{0} a^{2} \sqrt{\frac{4 V_{4}}{m}}$.

For the purposes of computation, we can use the fact that $F(\theta)$ is proportional to $\left(\frac{d \theta}{d \psi}\right)^{-1}$ and cancel this factor with a change of integration variables from $\theta$ to $\psi$. This results in

$$
\lambda_{n}^{l}=\frac{4 \pi}{\tau_{M}} \int_{0}^{\pi / 4} \frac{G_{n}^{l}[\theta(\psi)]}{\sin ^{2}(2 \psi)} d \psi
$$

which is much better suited to numerical integration than Eq. (23).

For the Maxwell gas, the placement of the zero eigenvalues is the same as in the hard-sphere case, meaning that particle number, momentum, and energy are conserved. Below we list eigenvalues for the first few values of $n$ and $l$, calculated using Eq. (25),

$$
\begin{aligned}
\lambda_{\mathbf{M}}^{\mathbf{0}}= & \frac{1}{\tau_{M}}(0, \quad 0, \quad-1.93796, \quad-2.90695, \\
& -3.57015, \quad-4.08046, \quad-4.49834, \quad \cdots),
\end{aligned}
$$

$$
\begin{aligned}
\lambda_{\mathbf{M}}^{\mathbf{1}}= & \frac{1}{\tau_{M}}(0, \quad-1.93796, \quad-2.90695, \quad-3.57015, \\
& -4.08046, \quad-4.49834, \quad-4.85402, \quad \cdots),
\end{aligned}
$$

and

$$
\begin{aligned}
\lambda_{\mathbf{M}}^{2}= & \frac{1}{\tau_{M}}(-2.90695, \quad-3.39144, \quad-3.90175, \\
& -4.33562, \quad-4.70728, \quad-5.03186, \quad \cdots) .
\end{aligned}
$$

The degeneracy of eigenvalues in $\lambda_{\mathbf{M}}^{\mathbf{0}}$ and $\lambda_{\mathbf{M}}^{\mathbf{1}}$ arises from the dependence of $G_{n}^{l}(\theta)$ on indices $n$ and $l$.

\section{HARD-SPHERE SIMULATION}

\section{A. Simulation method}

For a hard-sphere simulation, we begin by uniformly distributing $N$ spheres of diameter $\sigma$ within a cubic cell of volume $L^{3}$, with periodic boundary conditions, in such a way that no sphere overlaps with any other sphere. Specifically, if $\mathbf{r}_{i}$ is the position of the $i$ th sphere then for all $j \neq i$ we must have $\left|\mathbf{r}_{i}-\mathbf{r}_{j}\right|>\sigma$. We then assign each sphere a velocity $\mathbf{v}_{i}$ in such a way as to approximate a distribution function $\tilde{f}(\mathbf{v})$ $=f^{0}(\mathbf{v})\left[1+g_{0}(\mathbf{c})\right]$, where $g_{0}(\mathbf{c})$ is our desired initial perturbation. The choice of the initial perturbation will be discussed below.

Schematically, the simulation is carried out as follows: (a) For each pair of spheres, we compute the contact time $t_{i j}$, which is the time at which the spheres $i$ and $j$ will collide, should they follow straight line paths. This is found by the contact condition $\left|\mathbf{r}_{i}+\mathbf{v}_{i}\left(t_{i j}-t\right)-\mathbf{r}_{j}-\mathbf{v}_{j}\left(t_{i j}-t\right)\right|=\sigma$, where $t$ is the current time. We solve the contact condition for $t_{i j}$ and choose the smallest positive value for $t_{i j}-t$. (b) We then perform a search to find the smallest $t_{i j}$ value, $t^{*}$, which represents the time of the next collision. (c) All spheres are advanced to $t^{*}$ following straight-line paths according to their locations and velocities at $t$. (d) The colliding spheres have their velocities altered according to the kinematics of a purely elastic collision. (e) Then we update all values of $t_{i j}$ and proceed to step (b).

We implement periodic boundary conditions by adding two parts to the above algorithm: (1) In step (a), we calculate not one, but 27 values for $t_{i j}$, each representing sphere $j$ offset by $-L, 0$ or $L$ in the $x, y$ or $z$ direction. Elastic collision kinematics are also adjusted accordingly. (2) We calculate the time at which each particle will strike each wall and add this list of times to our search in step (b). If a wall collision occurs as the next soonest collision, that sphere is simply moved to the opposite side of the simulation cell.

\section{B. Data measurement}

As the simulation progresses, we have access to each $\mathbf{r}_{i}$ and each $\mathbf{v}_{i}$ at any iteration. With a typical particle number of $N=1000$, this is clearly a vast amount of information. Since our goal is observation of perturbation decay rates, the perturbation amplitudes $g_{n, l, m}$ are of particular interest. Let us see how we go about extracting these values from $\mathbf{r}_{i}$ and each $\mathbf{v}_{i}$.

Assume that we are given an arbitrary continuous distribution function $f(\mathbf{r}, \mathbf{v}, t)$ that exists in a volume $V$. We must first determine the number of particles present and their most probable velocity $v_{T}$ in equilibrium. These are given by $N$ $=\int d \mathbf{r} d \mathbf{v} f(\mathbf{r}, \mathbf{v}, t)$ and $v_{T}^{2}=\frac{2}{3 N} \int d \mathbf{r} d \mathbf{v} v^{2} f(\mathbf{r}, \mathbf{v}, t)$. In order to determine $g_{n, l, m}(t)$, we use the equation which defines the perturbations,

$$
f(\mathbf{r}, \mathbf{v}, t)=\frac{N}{V} \frac{1}{\pi^{3 / 2} v_{T}^{3}} e^{-c^{2}}\left(1+\sum_{n, l, m} \chi_{n, l, m}(\mathbf{c}) g_{n, l, m}(t)\right),
$$

where $\mathbf{c}=\frac{\mathbf{v}}{v_{T}}$. Using the orthogonality of the polynomials $\chi_{n, l, m}$, we can invert Eq. (29) to get an explicit formula for $g_{n, l, m}(t)$, 
$g_{n, l, m}(t)=-\pi^{3 / 4} \delta_{n, 0} \delta_{l, 0} \delta_{m, 0}+\frac{\pi^{3 / 2} v_{T}^{3}}{N} \int d \mathbf{r} d \mathbf{c} \chi_{n, l, m}^{*}(\mathbf{c}) f(\mathbf{r}, \mathbf{v}, t)$.

We now construct a phase space density function $\nu(\mathbf{r}, \mathbf{v}, t)$ from $\mathbf{r}_{i}(t)$ and $\mathbf{v}_{i}(t)$ by writing

$$
\nu(\mathbf{v}, t)=\sum_{i=1}^{N} \delta^{3}\left[\mathbf{r}-\mathbf{r}_{i}(t)\right] \delta^{3}\left[\mathbf{v}-\mathbf{v}_{i}(t)\right] .
$$

This phase space density function is used in place of $f(\mathbf{r}, \mathbf{v}, t)$ in Eq. (30). Explicitly, this is

$$
g_{n, l, m}(t)=-\pi^{3 / 4} \delta_{n, 0} \delta_{l, 0} \delta_{m, 0}+\frac{\pi^{3 / 2}}{N} \sum_{i=1}^{N} \chi_{n, l, m}^{*}\left(\frac{\mathbf{v}_{i}(t)}{v_{T}}\right) .
$$

With Eq. (32) we can measure the decay of perturbations as the system evolves. It is of interest to note that by construction, $g_{0,0,0}(t)=0, \quad g_{0,1,0}(t) \propto\left\langle c_{z}\right\rangle=0, \quad g_{0,1,1}(t) \propto\left\langle c_{x}\right\rangle-i\left\langle c_{y}\right\rangle=0$, $g_{0,1,-1}(t) \propto\left\langle c_{x}\right\rangle+i\left\langle c_{y}\right\rangle=0$, and $g_{1,0,0}(t) \propto v_{T}^{2}-\frac{2}{3}\left\langle v^{2}\right\rangle=0$. This enforces the idea that the quantities $N,\langle\mathbf{c}\rangle$, and $\left\langle c^{2}\right\rangle$ are not expressed as perturbations, and therefore are conserved.

\section{Decay of perturbations}

In the real-world hard-spheres problem, the time scale is set by $\frac{1}{\tau_{\mathrm{HS}}}=n_{0} \sigma^{2} \sqrt{4 \pi v_{T}^{2}}$ [see Eq. (15) and the definition of $\left.v_{T}\right]$. Measuring our eigenvalues in units of $\frac{1}{\tau_{\mathrm{HS}}}$ yields dimensionless eigenvalues that are universal to any hard-sphere system. We can compute the time scale for our simulations, using the formula

$$
\frac{1}{\tau_{\mathrm{HS}}}=\frac{N \sigma^{2}}{L^{3}} \sqrt{4 \pi \frac{2}{3 N} \sum_{i=1}^{N} v_{i}^{2}} .
$$

Note that events always occur at the same rate provided that time is measured in units of $\tau_{\mathrm{HS}}$. Because of this, and for convenience, we choose to normalize all velocities before the simulation begins such that $\frac{2}{3 N} \Sigma_{i=1}^{N} v_{i}^{2}=1$. Once this is done, the time scale can be found from $\frac{1}{\tau_{\mathrm{HS}}}=\left(N \sigma^{2} / L^{3}\right) \sqrt{4 \pi}$.

We can characterize the density by the unitless ratio $\rho$ $=\frac{N a^{3}}{L^{3}}$, where $a=\frac{\sigma}{2}$ is the radius of the particle. This number can be used to compare a given choice of $N, \sigma$, and $L$ to a real-world situation. Room temperature ideal gases have $\rho$ $\approx 10^{-3}$ while ultracold trapped gases have $\rho \approx 10^{-8}$. Simulations we have run up to this point have had $\rho \sim 10^{-5}$. Simulations with lower densities take considerably longer to complete, due to the dominance of wall collisions.

In order to observe decay to equilibrium, we must begin with a distribution that is not in equilibrium. This is accomplished by choosing the initial velocities of the spheres such that $g_{0}(\mathbf{c}) \neq 0$. We do not expect the specific distribution of initial velocities to have much effect on the evolution of the system, so we choose a form of $g_{0}(\mathbf{c})$ that gives large values for the lowest-order perturbation amplitudes. The initial condition used was
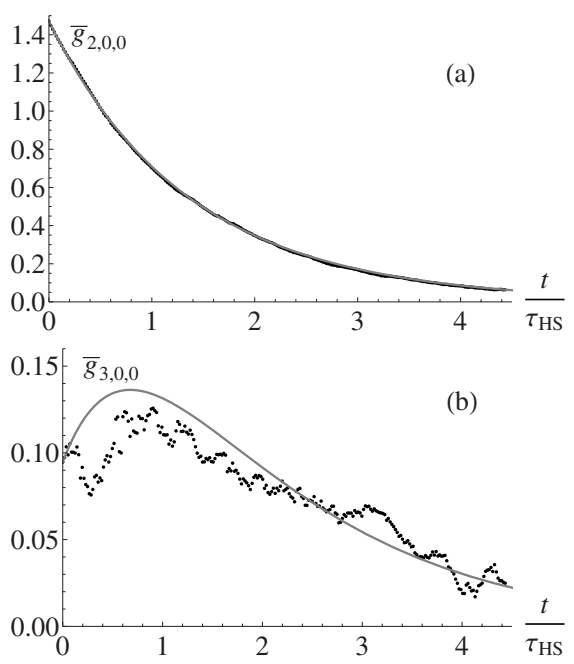

FIG. 1. Plots of (a) $\bar{g}_{2,0,0}(t)$ and (b) $\bar{g}_{3,0,0}(t)$ obtained from numerical simulation (dots) compared to calculated values (solid curve) obtained from Eq. (9) using the same initial distribution.

$$
g_{0}(\mathbf{c})=\alpha \chi_{2,0,0}(\mathbf{c})
$$

where $\alpha$ is a parameter used to set the magnitude of the perturbation. Choosing this form for the initial condition results in $g_{0}=\alpha(0,0,1,0,0, \ldots)$. In order to observe the exponential decay over the noise, $\alpha$ must be chosen as large as 0.5 or 0.75 . While this does not satisfy $g(\mathbf{c}) \ll 1$, we observed pure exponential decay with negligible nonlinear effects. However, some care must be taken, since too large a value for $\alpha$ will create regions where the distribution function $f(\mathbf{c})$ becomes negative.

As an example, consider the decay of a given perturbation amplitude $g_{n, l, m}$. In practice, the perturbation amplitudes $g_{n, l, m}$ from a single run are far too noisy to give any useful estimation of the eigenvalues. To compensate for this, we run up to 100 trials for a single set of parameters and store the data from each trial in a separate file. Later, a separate program parses these files and merges them according to

$$
\bar{g}_{n, l, m}=\frac{1}{M} \sum_{\beta=1}^{M} g_{n, l, m}^{\beta},
$$

where $M$ is the total number of trials included in the merge and $g_{n, l, m}^{\beta}$ indicates the data from the $\beta$ th trial. Examples of results obtained for $\bar{g}_{2,0,0}(t)$ and $\bar{g}_{3,0,0}(t)$ are shown in Fig. 1.

\section{Extraction of eigenvalues}

The functions $\chi_{n, l, m}$ are not the eigenfunctions of the hardsphere collision operator and therefore the quantities $\bar{g}_{n, l, m}(t)$ will not exhibit independent exponential decay. The true eigenfunctions for hard spheres are given by a linear combination of $\chi_{n, l, m}$ that is determined by the eigenvectors of the collision operator matrices $\mathbf{C}^{l}$. We perform a linear transformation on the $\bar{g}_{n, l, m}(t)$ to obtain the correct coefficients of expansion terms of the eigenfunctions of the hard-sphere collision operator, 


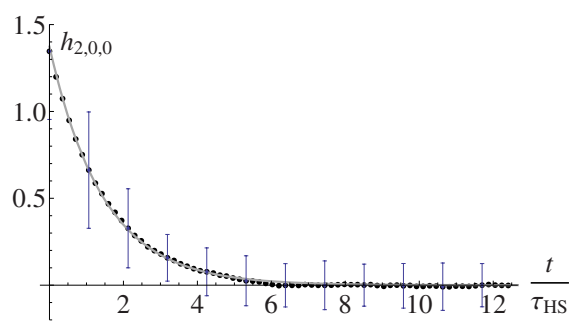

FIG. 2. (Color online) A plot of $h_{2,0,0}(t)$ as it evolves in the hard-spheres simulation. Error bars show the extent of the statistical error. Dots show values obtained from numerical simulation. The solid line is an exponential regression. Here $N=1000, \sigma=0.005$, and $L=2$. These data points come from the same data set used to obtain Fig. 1.

$$
h_{n, l, m}(t)=\sum_{n^{\prime}=0}^{\infty} \Phi_{n, n^{\prime}}^{l} \bar{g}_{n^{\prime}, l, m},(t),
$$

where $\boldsymbol{\Phi}^{l}$ is the matrix of eigenvectors of the collision matrix $\mathbf{C}^{l}$. The quantities $h_{n, l, m}(t)$ do exhibit exponential decay. A plot of $h_{2,0,0}$ vs $\frac{t}{\tau_{\mathrm{HS}}}$ is shown in Fig. 2 for $N=1000, \sigma$ $=0.005$ and $L=2$. A comparison of values of the first and second largest nonzero eigenvalues for the hard-sphere gas, obtained from theory and from the molecular dynamics simulation, are shown in Table I and are in good agreement.

\section{TRANSPORT COEFFICIENTS}

The coefficients of shear viscosity $\eta$ and thermal conductivity $\kappa$, in terms of dimensionless velocity coordinates, are given by $[9,10]$

$$
\eta=-\frac{4 n_{0} k_{B} T}{\pi^{3 / 2}} \int d \mathbf{c} e^{-c^{2}} c_{x} c_{y} \hat{C}_{\mathbf{c}}^{-1}\left[c_{y} c_{x}\right]
$$

and

$$
\kappa=-\frac{2 n_{0} k_{B}^{2} T}{m \pi^{3 / 2}} \int d \mathbf{c} e^{-c^{2}}\left(c^{2}-\frac{5}{2}\right) c_{x} \hat{C}_{\mathbf{c}}^{-1}\left[c_{x}\left(c^{2}-\frac{5}{2}\right)\right],
$$

where the function $\hat{C}_{\mathbf{c}}[g]$ is defined

$$
\begin{aligned}
\hat{C}_{\mathbf{c}}[g]= & \int d \mathbf{c}_{5} e^{-c_{5}^{2}} C\left(\mathbf{c}, \mathbf{c}_{5}\right) g\left(\mathbf{c}_{5}\right) \\
= & n_{0} v_{T} \frac{8}{\pi^{3 / 2}} \int d \mathbf{c}_{2} d \mathbf{c}_{3} d \mathbf{c}_{4} I(u, \theta) \delta^{3}\left(\mathbf{c}+\mathbf{c}_{2}-\mathbf{c}_{3}-\mathbf{c}_{4}\right) \\
& \times \delta\left(c^{2}+c_{2}^{2}-c_{3}^{2}-c_{4}^{2}\right) e^{-c_{2}^{2}}\left[g\left(\mathbf{c}_{3}\right)+g\left(\mathbf{c}_{4}\right)-g(\mathbf{c})\right. \\
& \left.-g\left(\mathbf{c}_{2}\right)\right] .
\end{aligned}
$$

In general, transport coefficients contain an integral of the type

$$
I=\int d \mathbf{c} e^{-c^{2}} A^{*}(\mathbf{c}) \hat{C}_{\mathbf{c}}^{-1}[A],
$$

where $\hat{C}_{\mathbf{c}}^{-1}[A]$, denotes the inverse operator $\hat{C}_{\mathbf{c}}^{-1}$ acting on function $A$. By expanding the function $A(\mathbf{c})$ in terms of the polynomials $\chi_{n, l, m}$, we can express this as

$$
I=\sum_{n, l, m} \sum_{n^{\prime}} A_{n, l, m}^{*}\left[\mathbf{C}^{l}\right]_{n, n^{\prime}}^{-1} A_{n^{\prime}, l, m},
$$

where $A_{n, l, m}$ are the expansion coefficients of the function $A(\mathbf{c})$ [the derivation of Eq. (41) from Eq. (40) is outlined in Appendix B]. For the shear viscosity, the function is $A(\mathbf{c})$ $=c_{x} c_{y}$ and the expansion coefficients are given by

$$
A_{n, l, m}=\frac{i \pi^{3 / 4}}{2 \sqrt{2}} \delta_{n, 0} \delta_{l, 2}\left(\delta_{m, 2}-\delta_{m,-2}\right) .
$$

The integral is equal to

$$
I=\frac{\pi^{3 / 2}}{4}\left[\mathbf{C}^{2}\right]_{0,0}^{-1}
$$

and so the transport coefficient is equal to

$$
\eta=-n_{0} k_{B} T\left[\mathbf{C}^{2}\right]_{0,0}^{-1} .
$$

Employing a similar procedure for $\kappa$ results in

$$
\kappa=-\frac{5 n_{0} k_{B}^{2} T}{2 m}\left[\mathbf{C}^{1}\right]_{1,1}^{-1} .
$$

For hard spheres, we can compute the inverse matrix elements numerically, and obtain $\left[\mathbf{C}^{2}\right]_{0,0}^{-1}=-0.898056 \tau_{\mathrm{HS}}$ and $\left[\mathbf{C}^{1}\right]_{1,1}^{-1}=-1.35926 \tau_{\mathrm{HS}}$. The transport coefficients for a hardsphere gas are then given by

TABLE I. Simulated dimensionless eigenvalues for several sets of parameters. In all cases, $L=2$.

\begin{tabular}{lcccc}
\hline \hline$N$ & $\sigma$ & $\rho$ & $\lambda_{2}^{0}$ & $\lambda_{3}^{0}$ \\
\hline 600 & 0.0025 & $9.37 \times 10^{-6}$ & $-0.68 \pm 0.10$ & $-0.92 \pm 0.10$ \\
700 & 0.00375 & $3.69 \times 10^{-5}$ & $-0.649 \pm 0.048$ & $-0.775 \pm 0.081$ \\
800 & 0.0025 & $1.25 \times 10^{-5}$ & $-0.682 \pm 0.072$ & $-0.92 \pm 0.21$ \\
1000 & 0.005 & $1.25 \times 10^{-4}$ & $-0.686 \pm 0.027$ & $-0.95 \pm 0.12$ \\
1200 & 0.0025 & $1.88 \times 10^{-5}$ & $-0.670 \pm 0.045$ & $-1.12 \pm 0.33$ \\
1200 & 0.005 & $1.50 \times 10^{-4}$ & $-0.669 \pm 0.017$ & $-0.99 \pm 0.12$ \\
Theory & & & -0.671227 & -0.911572 \\
\hline \hline
\end{tabular}




$$
\eta=(0.898056) \frac{1}{\sigma^{2}} \sqrt{\frac{m k_{B} T}{8 \pi}}
$$

and

$$
\kappa=(1.35926) \frac{5 k_{B}}{2 \sigma^{2}} \sqrt{\frac{k_{B} T}{8 \pi m}} .
$$

It is interesting to note that if we neglect the off-diagonal elements, we obtain the same numerical coefficients given in standard texts $[10,11]$ of $\frac{5 \sqrt{2}}{8}=0.8838835$ for $\eta$ and $\frac{15 \sqrt{2}}{16}$ $=1.325825$ for $\kappa$

The numerical coefficients computed from the inverse of the full matrix agree with the expected increase in the transport coefficients for hard spheres when off-diagonal contributions are included [9]. The power of this method is that once the elements of the collision matrix are known, transport coefficients can be computed quickly and accurately. This can be especially useful for collision matrices which are computed numerically from a scattering cross section and collision matrices resulting from phenomenological collision kernels.

\section{CONCLUSION}

We have computed the smallest nonzero eigenvalue of the collision operator for a hard-sphere gas containing 1000 particles. The value we obtain agrees with the theoretical prediction, to within the error induced by fluctuations due to the small number of particles in the simulation. We show that the off-diagonal elements of the collision operator make contributions to the transport coefficients which are not included in the standard derivations. Our method of analysis, in terms of moments of the perturbation function $g(\mathbf{c})$, could also be applied to many molecular dynamics systems.

\section{ACKNOWLEDGMENT}

The authors wish to thank the Robert A. Welch Foundation (Grant No. F-1051) for partial support of this work.

\section{APPENDIX A: CALCULATION OF THE MATRIX ELEMENTS $M_{n, n^{\prime}}^{l}$ FOR HARD SPHERES}

To compute Eq. (15), combine Eqs. (3) and (14) and split the resulting expression for $M(x, y ; t)$ into three parts, $M_{1}(x, y ; t), M_{2}(x, y ; t)$, and $M_{34}(x, y ; t)$, such that

$$
\begin{aligned}
M_{1}(x, y ; t)= & \int d \mathbf{c}_{1} d \mathbf{c}_{5} d \mathbf{c}_{2} d \mathbf{c}_{3} d \mathbf{c}_{4} G\left(x, y, t ; \mathbf{c}_{1}, \mathbf{c}_{2}, \mathbf{c}_{3}, \mathbf{c}_{4}, \mathbf{c}_{5}\right) \\
& \times \delta^{3}\left(\mathbf{c}_{1}-\mathbf{c}_{5}\right), \\
M_{2}(x, y ; t)= & \int d \mathbf{c}_{1} d \mathbf{c}_{5} d \mathbf{c}_{2} d \mathbf{c}_{3} d \mathbf{c}_{4} G\left(x, y, t ; \mathbf{c}_{1}, \mathbf{c}_{2}, \mathbf{c}_{3}, \mathbf{c}_{4}, \mathbf{c}_{5}\right) \\
& \times \delta^{3}\left(\mathbf{c}_{2}-\mathbf{c}_{5}\right),
\end{aligned}
$$

and

$$
\begin{aligned}
M_{34}(x, y ; t)= & \int d \mathbf{c}_{1} d \mathbf{c}_{5} d \mathbf{c}_{2} d \mathbf{c}_{3} d \mathbf{c}_{4} G\left(x, y, t ; \mathbf{c}_{1}, \mathbf{c}_{2}, \mathbf{c}_{3}, \mathbf{c}_{4}, \mathbf{c}_{5}\right) \\
& \times\left[\delta^{3}\left(\mathbf{c}_{3}-\mathbf{c}_{5}\right)+\delta^{3}\left(\mathbf{c}_{4}-\mathbf{c}_{5}\right)\right]
\end{aligned}
$$

where

$$
\begin{aligned}
G(x, y, t & \left.; \mathbf{c}_{1}, \mathbf{c}_{2}, \mathbf{c}_{3}, \mathbf{c}_{4}, \mathbf{c}_{5}\right) \\
= & n_{0} \sigma^{2} v_{T} \frac{1}{\pi^{5 / 2}[(1-x)(1-y)]^{3 / 2}} \\
& \times \delta^{3}\left(\mathbf{c}_{1}+\mathbf{c}_{2}-\mathbf{c}_{3}-\mathbf{c}_{4}\right) \delta\left(c_{1}^{2}+c_{2}^{2}-c_{3}^{2}-c_{4}^{2}\right) \\
& \times e^{-c_{1}^{2}-c_{2}^{2}-x c_{1}^{2} /(1-x)-y c_{5}^{2} /(1-y)+t \mathbf{c}_{1} \cdot \mathbf{c}_{5} /[(1-x)(1-y)]} \\
& \times \int_{0}^{2 \pi} d \phi e^{i t\left|\mathbf{c}_{1} \times \mathbf{c}_{5}\right| \cos \phi /[(1-x)(1-y)]}
\end{aligned}
$$

so that $M(x, y ; t)=-M_{1}(x, y ; t)-M_{2}(x, y ; t)+M_{34}(x, y ; t)$.

We will now work out the term $M_{1}(x, y ; t)$ in detail as an example. First to do the $\mathbf{c}_{5}$ integration. This results in

$$
\begin{aligned}
M_{1}(x, y ; t)= & n_{0} \sigma^{2} v_{T} \frac{2}{\pi^{3 / 2}[(1-x)(1-y)]^{3 / 2}} \int d \mathbf{c}_{1} d \mathbf{c}_{2} d \mathbf{c}_{3} d \mathbf{c}_{4} \\
& \times \delta^{3}\left(\mathbf{c}_{1}+\mathbf{c}_{2}-\mathbf{c}_{3}-\mathbf{c}_{4}\right) \delta\left(c_{1}^{2}+c_{2}^{2}-c_{3}^{2}-c_{4}^{2}\right) \\
& \times e^{-c_{1}^{2}-c_{2}^{2}-x c_{1}^{2} /(1-x)-y c_{1}^{2} /(1-y)+t c_{1}^{2} /[(1-x)(1-y)]} .
\end{aligned}
$$

Integration over $\mathbf{c}_{1}, \mathbf{c}_{2}, \mathbf{c}_{3}$, and $\mathbf{c}_{4}$ then gives (after some algebra)

$$
M_{1}(x, y ; t)=2 \pi^{2} n_{0} \sigma^{2} v_{T} \frac{\sqrt{2-x-y-t}}{(1-x y-t)^{2}} .
$$

$M_{2}(x, y ; t)$ and $M_{34}(x, y ; t)$ can also be computed and are given by

$$
M_{2}(x, y ; t)=2 \pi^{2} n_{0} \sigma^{2} v_{T} \sqrt{2-x-y-t}
$$

and

$$
M_{34}(x, y ; t)=4 \pi^{2} n_{0} \sigma^{2} v_{T} \frac{\sqrt{2-x-y-t}}{1-x y-t+\frac{t^{2}}{4}} .
$$

Recombining these three terms results in

$$
\begin{aligned}
M(x, y ; t)= & 2 \pi^{2} n_{0} \sigma^{2} v_{T} \sqrt{2-x-y-t} \\
& \times\left(-\frac{1}{(1-x y-t)^{2}}-1+\frac{2}{1-x y-t+\frac{t^{2}}{4}}\right) .
\end{aligned}
$$

The fact that $M(x, y ; t)$ is symmetric in $x$ and $y$ implies that the collision matrix is symmetric and that the function $C\left(\mathbf{c}_{1}, \mathbf{c}_{5}\right)$ is symmetric in $\mathbf{c}_{1}$ and $\mathbf{c}_{5}$. Using generalizations of the geometric series given by expressions like $(1-A-B)^{-\nu}$ $=\sum_{j=0}^{\infty} \sum_{k=0}^{\infty} \frac{\Gamma(\nu+j+k)}{\Gamma(\nu) j ! k !} A^{j} B^{j}$, We re-expand $M(x, y ; t)$ in powers of $x, y$, and $t$ and rewrite the coefficient using $2 \pi^{2} n_{0} \sigma^{2} v_{T}=\frac{\pi^{3 / 2}}{\tau_{\mathrm{HS}}}$. We then obtain Eq. (15). 


\section{APPENDIX B: TRANSFORMATION OF THE TRANSPORT COEFFICIENT INTEGRAL}

In this appendix, we show the steps to go from Eq. (40) to Eq. (41). Define $B(\mathbf{c})$ as the function for which

$$
A(\mathbf{c})=\hat{C}_{\mathbf{c}}[B]
$$

If we expand both $A(\mathbf{c})$ and $B(\mathbf{c})$ in terms of the polynomials $\chi_{n, l, m}(\mathbf{c})$, this becomes

$$
\sum_{n^{\prime \prime}, l^{\prime \prime}, m^{\prime \prime}} A_{n^{\prime \prime}, l^{\prime \prime}, m^{\prime \prime}} \chi_{n^{\prime \prime}, l^{\prime \prime}, m^{\prime \prime}}(\mathbf{c})=\sum_{n^{\prime}, l^{\prime}, m^{\prime}} B_{n^{\prime}, l^{\prime}, m^{\prime}} \hat{C}_{\mathbf{c}}\left[\chi_{n^{\prime}, l^{\prime}, m^{\prime}}\right] .
$$

Multiplying by $e^{-c^{2}} \chi_{n, l, m}^{*}(\mathbf{c})$ and integrating over $\mathbf{c}$, we obtain

$$
A_{n, l, m}=\sum_{n^{\prime}, l^{\prime}, m^{\prime}} B_{n^{\prime}, l^{\prime}, m^{\prime}} \int d \mathbf{c} e^{-c^{2}} \chi_{n, l, m}^{*}(\mathbf{c}) \hat{C}_{\mathbf{c}}\left[\chi_{n^{\prime}, l^{\prime}, m^{\prime}}\right] .
$$

Comparing this to Eqs. (7) and (39) we see that

$$
\int d \mathbf{c} e^{-c^{2}} \chi_{n, l, m}^{*}(\mathbf{c}) \hat{C}_{\mathbf{c}}\left[\chi_{n^{\prime}, l^{\prime}, m^{\prime}}\right]=C_{n, n^{\prime}}^{l} \delta_{l, l^{\prime}} \delta_{m, m^{\prime}}
$$

and thus if the expansion coefficients $A_{n, l, m}$ are known, we determine $B_{n, l, m}$ as the values which satisfy

$$
A_{n, l, m}=\sum_{n^{\prime}} C_{n, n^{\prime}}^{l} B_{n^{\prime}, l, m} .
$$

This is easily solved if we treat $C_{n n^{\prime}}^{l}$ as a matrix $\mathbf{C}^{l}$, but we know that $\mathbf{C}^{0}$ and $\mathbf{C}^{1}$ are noninvertible. However, we can still find a solution to Eq. (B5) provided that we use a function $A(\mathbf{c})$ for which $A_{0,0, m}=0, A_{1,0, m}=0$, and $A_{0,1, m}=0$. In these cases, we trivially solve the zero rows and remove them from the matrix. The remaining matrix is then invertible and can be used to find the remaining $B_{n, l, m}$. We therefore write

$$
B_{n, l, m}=\sum_{n^{\prime}}\left[C^{l}\right]_{n, n^{\prime}}^{-1} A_{n^{\prime}, l, m},
$$

where the above process and condition on $A_{n, l, m}$ are implied. We now turn back to the integral expression $I$ in Eq. (40) and substitute $B(\mathbf{c})$ for $\hat{C}_{\mathbf{c}}^{-1}[A]$. Then expand the function $A(\mathbf{c})$ and $B(\mathbf{c})$ in terms of $\chi_{n, l, m}(\mathbf{c})$,

$$
I=\int d \mathbf{c} e^{-c^{2}} \sum_{n, l, m} A_{n, l, m}^{*} \chi_{n, l, m}^{*}(\mathbf{c}) \sum_{n^{\prime}, l^{\prime}, m^{\prime}} B_{n^{\prime}, l^{\prime}, m^{\prime}} \chi_{n^{\prime}, l^{\prime}, m^{\prime}}(\mathbf{c}) .
$$

We quickly see that

$$
I=\sum_{n, l, m} A_{n, l, m}^{*} B_{n, l, m},
$$

and using the above expression for $B_{n, l, m}$ we have

$$
I=\sum_{n, l, m} \sum_{n^{\prime}} A_{n, l, m}^{*}\left[C^{l}\right]_{n, n^{\prime}}^{-1} A_{n^{\prime}, l, m} .
$$

[1] C. S. Wang Chang and G. E. Uhlenbeck, University of Michigan, Ann Arbor, Report No. M999, 1952. See Studies in Statistical Mechanics, edited by J. de Boer and G. E. Uhlenbeck (North-Holland, Amsterdam, 1970), Vol. V, Chap. III.

[2] O. O. Jenssen, Phys. Norv. 6, 179 (1972).

[3] C. Cercignani, Theory and Application of the Boltzmann Equation (Elsevier, New York, 1975), pp. 180-186.

[4] B. Shizgal, Can. J. Phys. 62, 97 (1984).

[5] K. E. Gibble and A. Gallagher, Phys. Rev. A 43, 1366 (1991).

[6] J. E. M. Haverkort, J. P. Woerdman, and P. R. Berman, Phys. Rev. A 36, 5251 (1987).

[7] S. Kryszewski and G. Nienhuis, J. Phys. B 22, 3435 (1989).

[8] R. F. Snider, Phys. Rev. A 33, 178 (1986).

[9] S. Chapman and T. G. Cowling, Mathematical Theory of Non-
Uniform Gases (Cambridge University Press, Cambridge, 1970).

[10] L. E. Reichl, A Modern Course in Statistical Physics (Wiley, New York, 1998), pp. 704-710.

[11] F. Reif, Fundamentals of Statistical and Thermal Physics (McGraw-Hill, New York, 1965), p. 545.

[12] James A. McLennan, Introduction to Non-Equilibrium Statistcal Mechanics (Prentice Hall, New York, 1989).

[13] S. Kryszewski and J. Gondek, Phys. Rev. A 56, 3923 (1997).

[14] P. R. Berman, J. E. M. Haverkort, and J. P. Woerdman, Phys. Rev. A 34, 4647 (1986).

[15] I. Kuscer and M. M. R. Williams, Phys. Fluids 10, 1922 (1967). 